\title{
LOCALLY FINITE AND SOLVABLE SUBGROUPS OF SFIELDS
}

\author{
R. J. FAUDREE ${ }^{1}$
}

In this paper we will determine all locally finite subgroups of a sfield and give a criterion for determining when the group algebra of an ascending solvable torsion free group has a quotient sfield.

Notation And Definitions. A group $G$ has property $\mathrm{E}$ if it can be embedded in a sfield $D$ and property $E E$ if every automorphism of the group $G$ can be extended to be an automorphsim of $D$. For a more complete discussion see [5].

The result on locally finite groups depends mainly on a result of Amitsur (see [1]). A complete discussion of the following notation can be found in his paper.

$\pi$ will denote the set of all primes and $\pi_{1}$ the set of all odd primes $p$ such that 2 has odd order $\bmod p$. Let $m$ and $r$ be relatively prime integers. Put $s=(r-1, m), t=m / s$ and $n=$ minimal integer satisfying $r^{n} \equiv 1 \bmod m$. Denote by $G_{m, r}$ a group generated by two elements $A$ and $B$ satisfying $A^{m}=1, B^{n}=A^{t}$ and $B A B^{-1}=A^{r}$. Denote by $G_{m, r}^{\infty}$ a group $G$ which has a countable ascending tower of subgroups $\left\{H_{i}: 0 \leqq i<\infty\right\}$ such that $G=\bigcup_{i=1}^{\infty} H_{i}$ and each $H_{i}$ is isomorphic to $G_{m_{i}, r_{i}} . T^{*}, O^{*}, I^{*}$ will denote the binary tetrahedral, octahedral and icosahedral groups.

Let $p$ be a fixed prime dividing $m$.

$\alpha=\alpha_{p}$ is the highest power of $p$ dividing $m$.

$\eta_{p}$ is the minimal integer satisfying $r^{\eta_{p}} \equiv 1 \bmod \left(m p^{-\alpha}\right)$.

$\mu_{p}$ is the minimal integer satisfying $r^{\mu_{p}} \equiv p^{\mu^{\prime}} \bmod \left(m p^{-\alpha}\right)$ for some integer $\mu^{\prime}$.

$\delta_{p}$ is the minimal integer such that $p^{\delta_{p}} \equiv 1 \bmod \left(m p^{-\alpha}\right)$.

$\delta_{p}^{\prime}=\mu_{p} \delta_{p} / \eta_{p}$

Condition C. Integers $m$ and $r$ satisfy Condition $C$ if either

(I) $(n, t)=(s, t)=1$, or

(II) $n=2 n^{\prime}, m=2^{\alpha} m^{\prime}, s=2 s^{\prime}$ where $\alpha \geqq 2, m^{\prime}, s^{\prime}$ and $n^{\prime}$ are odd integers; $(n, t)=(s, t)=2$ and $r \equiv-1 \bmod 2^{\alpha}$. And either

(III) $n=s=2$ and $r \equiv-1(\bmod m)$, or

(IV) for every $q / n$ there exists a prime $p \mid m$ such that $q \nmid \eta_{p}$ and that either

Received by the editors January 6, 1968 .

${ }^{1}$ In partial support by NSF Grant GP 7029. The author would like to thank the referee for his helpful comments. 
(1) $p \neq 2$ and $\left(q,\left(p^{\delta^{\prime}}-1\right) / s\right)=1$, or

(2) $p=q=2$, (II) holds and $m / 4 \equiv \delta_{2}^{\prime} \equiv 1(\bmod 2)$.

Amitsur proved the following (see [1]):

Theorem 1. A finite group has property $\mathrm{E}$ if and only if $G$ is isomorphic to one of the following groups:

(i) cyclic group,

(ii) $G_{m, r}$ where $m$ and $r$ satisfy Condition $\mathrm{C}$,

(iii) $T^{*} \times G_{m, r}$ where $G_{m, r}$ is either cyclic of order $m$ or of type (2), $\left(G,\left|G_{m, r}\right|\right)=1$ and $p \mid m$ implies $p$ in $\pi_{1}$,

(iv) $O^{*}$ and $I^{*}$.

We will prove the following generalization of Amitsur's Theorem.

Theorem 2. Let $G$ be a locally finite group. The following are equivalent:

(1) G has property E,

(2) G has property EE,

(3) $G$ is isomorphic to

(i) a subgroup of $\prod_{p \in \pi} Z\left(p^{\infty}\right)$,

(ii) $G_{m, r}^{\infty}$ where $m_{i}$ and $r_{i}$ satisfy Condition $\mathrm{C}$,

(iii) $T^{*} \times H$ where either (a) $H$ is a subgroup of $\prod_{p \in \pi_{1}} Z\left(p^{\infty}\right)$, (b) $H=G_{m, r}^{\infty}$ where $\left(\left|G_{m_{i}, r_{i}}\right|, 6\right)=1, p \mid m_{i}$ implies $p$ is in $\pi_{1}$ and $m_{i}$ and $r_{i}$ satisfy Condition $\mathrm{C}$,

(iv) $O^{*}$ and $I^{*}$.

Theorem 2 for countable locally finite groups was proved in [6]. Thus to prove Theorem 2 it is sufficient to show that any locally finite subgroup of a sfield is countable.

Let $G$ be a locally finite group which can be embedded in a division ring. If $S$ is a subset of $G$ then $\langle S\rangle$ will denote the subgroup of $G$ generated by $S$.

Lemma 1. If $G$ has a subgroup isomorphic to $O^{*}$ or $I^{*}$ then $G$ is isomorphic to $O^{*}$ or $I^{*}$.

Proof. $O^{*}$ and $I^{*}$ are the only groups satisfying Theorem 1 which are not solvable of length 3 or less.

In the remaining lemmas it will be assumed that $G$ has no subgroups isomorphic to $O^{*}$ or $I^{*}$.

LEMma 2. If $G$ has $T^{*}$ as a subgroup then there is a subgroup $H$ of $G$ such that $G=T^{*} \times H$ and every finite subgroup of $H$ satisfies (I) of Condition $\mathrm{C}$.

Proof. Let $R=C_{G}\left(T^{*}\right)$. Let $H=\left\{x \in C_{G}\left(T^{*}\right)|| x \mid\right.$ is odd $\}$. If 
$x, y \in H$ and $g \in G$, then $\left\langle x, y, g, T^{*}\right\rangle=T^{*} \times S$ where $S$ is a subgroup of odd order satisfying (I) of Condition C by Theorem 1. $\left|Z\left(T^{*}\right)\right|=2$, thus $x, y \in S$. Therefore $H$ is a subgroup. Also $g=t \cdot s$ with $t \in T^{*}$, $s \in S \subseteq H$. Hence $G=T^{*} \times H$.

A factor of a group $G$ is a quotient group of a subgroup of $G$. The rank of an abelian group $G$ will be $k$ if it has an elementary abelian factor of order $p^{k}$ for some prime $p$ but no elementary abelian factor of order $p^{k+1}$ for some prime $p$. The derived factors of a group $G$ are the quotient groups $G_{i} / G_{i+1}$ where $G_{i}$ is the $i$ th derived group.

LEмма 4. If $G$ is a locally finite group embeddable in a sfield then $G \cong K \times H$ where $K$ is a finite group and $H$ is solvable of length $\leqq 2$ and each derived factor of $K$ is of rank $\leqq 2$.

Proof. By Lemmas 1,2 and Theorem $1, G=K \times H$ with $K \cong O^{*}$, $I^{*}$ or $T^{*}$ and every finite subgroup of $H$ is metacyclic. Since a metacyclic group is solvable of length $\leqq 2$ and each derived factor is of rank $\leqq 2$, the same is true of $H$.

LEMma 5. Let $G$ be a solvable group of derived length $n$. If for some $k$ each of the derived factors has rank at most $k$, then $G$ is countable.

Proof. If $A$ is an uncountable abelian group then it does not have finite rank. Thus $G_{i} / G_{i+1}$ is countable for each $i$ and hence $G$ is countable.

Theorem 2 is a consequence of Lemmas 4 and 5 .

It follows that in a sfield there is a maximal locally finite subgroup and it is countable.

If $G$ is a finite subgroup of a sfield $D$ and generates $D$ then the automorphism group of $D$ is determined by the automorphism group of $G$ modulo the inner automorphism group of $D$ (see [7]). It would be interesting to know if this is also true for a locally finite group.

Before proving the next theorem we will give some more definitions and notation.

A group $G$ is ascending solvable if $G$ has an ascending normal series such that each factor is abelian [9, p. 163]. If $K$ is a sfield and $G$ a group, then $K[G]$ will denote the group algebra of $G$ over $K$. If $\theta$ is an automorphism of $K$ and $x$ an indeterminate over $K, K[x, \theta]$ will denote the Ore polynomial ring in $x$ over $K$ determined by $\theta$ (see [11]). A ring $R$ is regular if it has no divisors of zero and if for elements $a$ and $b$ in $R$ there are nonzero elements $a_{1}, b_{1}, a_{2}$ and $b_{2}$ in $R$ such that $a a_{1}=b b_{1}$ and $a_{2} a=b_{2} b . F$ is a quotient sfield of $R$ if $R$ is a subring of the sfield $F$ and for any $f$ in $F, f=r_{1} r_{2}^{-1}=r_{3}^{-1} r_{4}$ for $r_{i}$ in $R(1 \leqq i \leqq 4)$. 
The above are connected by the following proposition proved by Asano [2].

Proposition 1. A ring $R$ has a quotient sfield if and only if $R$ is a regular ring. The quotient sfield is unique up to isomorphism. Any automorphism $\theta$ of $R$ can be extended uniquely to $F$.

The following result was proved by Ore [11]:

Proposition 2. $K[x ; \theta]$ is a regular ring.

The quotient sfield of $K[x ; \theta]$ will be denoted by $K(x ; \theta)$. If the group algebra $K[G]$ is a regular ring, its quotient sfield will be denoted by $K(G)$.

The following is a special case of a theorem of Bovdi [3]:

Proposition 3. If $G$ is an ascending solvable group and $K$ a sfield, then the group algebra $K[G]$ has a quotient sfield if and only if $K[G]$ has no divisors of zero.

We will give a criterion to determine when the group algebra has no divisors of zero. The following definition and proposition is due to Ore $[10]$.

Let $R$ be a regular ring and $A=\left(a_{i j}\right)$ be an $n \times n$ matrix with coefficients in $R$. We now give a determinant function $\mid \|$ for $A$.

If $n=1$, define $\mid A \|=a_{11}$. If $n=2$, define $\mid A \|=a_{11} A_{22}-a_{21} A_{12}$ where $A_{22}$ and $A_{12}$ have the property that $a_{12} A_{22}=a_{22} A_{12}$. For $n$ define $\mid A \|=a_{11} A_{1}{ }^{(1)}+a_{21} A_{1}{ }^{(2)}+\cdots+a_{n 1} A_{1}{ }^{(n)}$ where the $A_{1}{ }^{(j)}$ are a set of solutions to the homogeneous equations

$$
\begin{aligned}
& a_{12} A_{1}^{(1)}+a_{22} A_{1}^{(2)}+\cdots+a_{n 2} A_{1}^{(n)}=0 \\
& \vdots \\
& a_{1 n} A_{1}^{(1)}+a_{2 n} A_{1}^{(2)}+\cdots+a_{n n} A_{1}^{n}=0 .
\end{aligned}
$$

The function ||| has the following important property:

Proposition 4. The linear system

$$
\sum_{i=1}^{n} x_{i} a_{i j}=0 \quad(1 \leqq j \leqq n) \quad a_{i j} \in R
$$

has a nontrivial solution if and only if $\left|a_{i j}\right| \mid=0$.

THEOREM 3. Let $G$ be an ascending solvable group with an ascending normal series $\left\{H_{i}\right\}_{i=0}^{\alpha}$ with $H_{i+1} / H_{i}=\left\langle x_{i+1} \cdot H_{i}\right\rangle$. Let $n_{i+1}$ be the order of $x_{i+1} \bmod H_{i}$ and let $\theta_{i+1}$ be the automorphism of $K\left[H_{i}\right]$ induced by $x_{i+1} . K[G]$ has a quotient sfield if and only if for each $i$ that $n_{i+1}$ is 
finite and for all $n_{i+1}$ tuples $\left(d_{0}, \cdots, d_{n_{i+1}}\right)$ of elements of $K\left[H_{i}\right]$, $\left|a_{k j}\right| \mid \neq 0$ where

$$
\begin{aligned}
a_{k j} & =\theta_{i+1}^{j}\left(d_{k-j}\right) \quad \text { if } k \geqq j, \\
& =\theta_{i+1}^{j}\left(d_{n_{i+1+k-j}}\right) x_{i+1}^{n_{i+1}} \quad \text { if } k<j .
\end{aligned}
$$

Proof. By Proposition 3 it is sufficient to show that $K[G]$ has no divisors of zero. Assume that $K\left[H_{i}\right]$ has no divisors of zero for all $i<j \leqq \alpha$. If $j$ is a limit ordinal then $K\left[H_{j}\right]$ has no divisors of zero. If $j$ is not a limit ordinal then there are two possibilities, either $x_{j}$ has infinite order $\bmod H_{j-1}$ or finite order $\bmod H_{j-1}$. In the first case it is obvious that $K\left[H_{j}\right]$ has no divisors of zero.

Therefore we are left with considering the case of $G$ and $H$ groups with $H$ normal in $G, G / H=\langle x \cdot H\rangle$ where $x$ has order $n \bmod H$ and $K[H]$ has no divisors of zero. Let $\theta$ be the automorphism of $K[H]$ induced by $x$. Every element of $K[G]$ can be written uniquely in the form $\sum_{i=0}^{n-1} d_{i} x^{i}$ for $d_{i}$ in $K[H]$. Let $w=\sum_{i=0}^{n-1} d_{i} x^{i}$ be a fixed element of $K[G]$. If $w$ is a divisor of zero, then there is a $y=\sum_{i=0}^{n-1} y_{j} x^{j}$ with $y_{j}$ in $K[H]$ such that $y w=0$. Thus

$$
\sum_{j=0}^{n-1} y_{j}\left(x^{j} w\right)=\sum_{j=0}^{n-1} y_{j}\left(\sum_{i=0}^{n} \theta^{j}\left(d_{i}\right) x^{i+j}\right)=0 .
$$

This breaks down into the following system of $n$-equations in the unknowns $y_{i}$.

$$
\begin{gathered}
y_{0} d_{0}+y_{1} \theta\left(d_{n-1}\right) x^{n}+\cdots+y_{n-1} \theta^{n-1}\left(d_{1}\right) x^{n}=0 \\
y_{0} d_{1}+y_{1} \theta\left(d_{0}\right)+y_{n-1} \theta^{n-1}\left(d_{2}\right) x^{n}=0 \\
\vdots \\
\dot{\cdot} \\
v_{0} d_{n-1}+y_{1} \theta\left(d_{n-2}\right)+\cdots+y_{n-1} \theta^{n-1}\left(d_{0}\right)=
\end{gathered}
$$

Application of Proposition 4 completes the proof.

The determinant given in Theorem 3 is very complicated. For $n=2$ or 3 we will give a simpler form of the same expression. This will be done in the next two lemmas.

Lemma 6. Let $K$ be a sfield $0 \neq b \in K$ and $\theta$ an automorphism of $K$ such that $\theta(b)=b$ and $\theta^{n}$ is the same automorphism of $K$ as the automorphism induced by $b$ in $K$. Consider the ring $K[x ; \theta]$. The following are equivalent.

(1) $x^{n}-b$ is irreducible.

(2) $\left(x^{n}-b\right)$ is a prime ideal. 
(3) $K[x ; \theta] /\left(x^{n}-b\right)$ is a sfield.

Conditions (1), (2) and (3) imply that $\left(x^{n}-b\right)$ is maximal but the converse is not true.

Proof. $K[x ; \theta] /\left(x^{n}-b\right)$ is a sfield if and only if it has no divisors of zero [8, p. 158]. $x^{n}-b$ reducible implies that $\left(x^{n}-b\right)$ is not prime so (2) implies (1). If $K[x ; \theta] /\left(x^{n}-b\right)$ has no divisors of zero then $x^{n}-b$ is prime so (3) implies (2). If $x^{n}-b$ is irreducible and $f(x)$ is not in $\left(x^{n}-b\right)$, then there are elements $f_{1}(x)$ and $g_{1}(x)$ in $K[x ; \theta]$ such that $f(x) f_{1}(x)+\left(x^{n}-b\right) g_{1}(x)=1$ (see [11]). Thus $f(x)$ has a right inverse $\left(\bmod \left(x^{n}-b\right)\right)$ so $(1)$ implies $(3)$.

Since $K[x ; \theta]$ is a principal ideal ring (see [11]), certainly $\left(x^{n}-b\right)$ prime implies that $\left(x^{n}-b\right)$ is maximal.

Let $\epsilon$ be a primitive 4 th root of unity and $x$ a transcendental over $Q$, the rational numbers. Let $F=Q(\epsilon, x)$, the field obtained by adjoining $\epsilon$ and $x$, and let $\theta$ be the automorphism of $F$ determined by $\epsilon \rightarrow \epsilon$ and $x \rightarrow \epsilon x$. Consider the ring $F[y ; \theta]$ and the ideal $\left(y^{4}-x^{4}\right)$. Direct calculation verifies that $\left(y^{4}-x^{4}\right)$ is maximal but not prime.

LemMA 7. With the same hypothesis as in Lemma $6, b \neq \theta^{n-1}(a) \theta^{n-2}(a)$ . . $\theta(a) a$ for all $a$ in $K$ is a necessary condition for $x^{n}-b$ to be irreducible polynomial in $K[x ; \theta]$. For $n=2$ or 3 it is also sufficient.

Proof. Let $N(a)=\theta^{n-1}(a) \cdots \theta(a) \cdot a$. If $b=N(a)$, then

$$
\begin{aligned}
x^{n}-b= & \left(x^{n-1}+\theta^{n-1}(a) x^{n-2}+\cdots\right. \\
& \left.+N(a) \theta(a)^{-1} a^{-1} x+N(a) a^{-1}\right) \cdot(x-a) .
\end{aligned}
$$

Thus the condition is necessary. If $n=2$ and $x^{2}-b=(x-c)(x-a)$ for $a$ and $c$ in $K$, then $c a=-b$ and $-c-\theta(a)=0$. Thus $b=\theta(a) a$. If $n=3$ and $x^{3}-b=\left(x^{2}+d x+c\right)(x-a)$ for $a, b$ and $c$ in $K$, then just in the case $n=2$ one can verify that $b=\theta^{2}(a) \theta(a) a$.

With these lemmas it is easily seen that Theorem 3 can be modified to give

THEOREM 4. Let $G$ be an ascending solvable group with an ascending normal series $\left\{H_{i}\right\}_{i=0}^{\alpha}$ with $H_{i+1} / H_{i}=\left\langle x_{i+1} \cdot H_{i}\right\rangle$. Let $n_{i+1}$ be the order of $x_{i+1} \bmod H_{i}$ and let $\theta_{i}$ be the automorphism of $K\left[H_{i}\right]$ induced by $x_{i+1}$. If $n_{i+1}=2,3$ or $\infty$ for each $i$, then $K[G]$ has a quotient sfield if and only if for each $i$ that $n_{i+1}$ is finite, $x_{i+1}^{n_{i}+1} \neq \theta_{i+1}^{n_{i+1}-1}(d) \cdots \theta(d) \cdot d$ for all $d$ in $K\left(H_{i}\right)$.

The theory developed in Theorem 4 would be included in the results of [5] if the group algebra of an ascending solvable group $G$ having no divisors of zero implies that $G$ has a normal series such 
that every factor is infinite cyclic. This is not true. Consider the group $G=\left\langle b_{1}, b_{2}, c \mid c^{-1} b_{i} c=b_{i}{ }^{-1}(1 \leqq i \leqq 2), b_{1}^{-1} b_{2}{ }^{-1} b_{1} b_{2}=c^{4}\right\rangle . G$ does not have a normal series such that each factor is infinite cyclic (see [4]) but direct calculation verifies that it satisfies Theorem 4.

\section{REFERENCES}

1. S. A. Amitsur, Finite subgroups of division rings, Trans. Amer. Math. Soc. 80 (1955), 361-386.

2. K. Asano, Uber die Quotientenbildung von Schiefringen, J. Math. Soc. Japan 1 (1949), 73-78.

3. A. A. Bovdi, Imbedding of crossed products in fields, Soviet Math. Dokl. 2 (1963), 1157-1159.

4. J. F. Bowers, On composition series of polycyclic groups, J. London Math. Soc. 35 (1960), 433-444.

5. R. J. Faudree, Subgroups of the multiplicative group of a division ring, Trans. Amer. Math. Soc. 124 (1966), 41-48.

6. - Embedding theorems for ascending nilpotent groups, Proc. Amer. Math. Soc. 18 (1967), 148-154.

7. - A Atomorphism groups of finite subgroups of division rings, Pacific J. Math. 26 (1968), 59-65.

8. N. Jacobson, Structure of rings, Amer. Math. Soc. Colloq. Publ., Vol. 37, Amer. Math. Soc., Providence, R. I., 1956.

9. A. G. Kurosh, Theory of groups, Vol. II, Chelsea, New York, 1960.

10. O. Ore, Linear equations in non-commutative fields, Ann. of Math. 32 (1931), 463-477. 508.

11. Theory of non-commutative polynomials, Ann. of Math. 34 (1933), 480-

UNIVERSITY OF ILLINOIS 